\title{
BIOLOGIA DEL CAMARON DE AGUA DULCE PALAEMONETES ZARIQUIEYI SOLLAUD, 1939 (CRUSTACEA: DECAPODA: PALAEMONIDAE)
}

\author{
A. Sanz Brau \\ Dpto. de Ecología. Facultad de Ciencias Biológicas. Universidad de Valencia.
}

Palabras clave: Palaemonetes zariquieyi, life history, growth, reproduction.

\author{
ABSTRACT \\ LIFE HISTORY OF THE FRESHWATER SHRIMP PALAEMONETES ZARIQUIEYI SOLLAUD, \\ 1939 (CRUSTACEA: DECAPODA: PALAEMONIDAE)
}

The life history of Palaemonetes zariquieyi Sollaud, 1939 was studied in three different places, with different ecological conditions, during two consecutive years. Place A (Castellón) shows a constant water temperature throughout year. Non great changes appear in the population. But in places B (Sagunto)and C (Torrente)a 1-year life cycle can be appreciate with a breeding season centered in june. But there are some individuals thai can survive their second winter of life. There appear females which can breed a second time, depending of the partial maturation of their gonads. The number of eggs carried by the females show a linear relation with the total lenght of the shnmps. The size reached by the females is significantly larger than that ofthe males. The sex ratio $1: 1$ in places $\mathrm{A}$ and $\mathrm{C}$, but 3:2 in place $\mathrm{B}$, that is possibly a result ofthe selective predation ofthe eels on ovigerous females.

\section{INTRODUCCION}

El camarón Palaernonetes zariquieyi Sollaud, 1939, es una especie endémica de la península Ibérica (Holthuis, 1978). Habita en aguas continentales de escasa o nula corriente, sitas en la franja costera entre el delta del río Ebro y el cabo de San Antonio. A pesar de tan restringida área de dispersión, las características ecológicas de las aguas en cada una de sus localidades son a veces muy distintas entre si, tanto en lo que se refiere a sus aspectos físico-químicos, como a los biológicos (Sanz, 1980; 1983).

Su biología es poco conocida, puesto que solo aparecen en la bibliografía citas referentes a algùnas de sus localidades (Zariquiey, 1946; 1968; Margalef , 1953), aspectos de sus características morfológicas distintivas, además de su descripción en la que Sollaud (1939) incluía algunos datos sobre las aguas donde habita y razonaba su supuesta evolución a partir de Palaernonetes varians (Leach, 1814).

En este trabajo se aborda el estudio de la biología de este camarón en tres localidades con características diferentes entre si, y que se estiman son típicas y representativas de las restantes en las que habita.

Limnética 2: 293-304 (1986)

O Asociación Española de Limnologia, Madrid. Spain

\section{LOCALIDADES DE ESTUDIO}

La tabla 1 y la figura 1 recogen las características fisico-químicas de las localidades a lo largo del periodo de estudio.

El punto A (Castellón) es un surgente («ullal») con aguas totalmente transparentes, en donde las condiciones ambientales son muy constantes a lo largo del año, especialmente la temperatura. La única vegetación presente es la graminea Panicum repens que crece en los márgenes y sobre la que vive P. zariquieyi, conjuntamente con Dugastella valentina, Crustacea Decapoda también endémico del levante ibérico y donde es dominante, puesto que la relación entre ambas especies es de 10:1.

El punto B (Sagunto) corresponde a una serie de canales de una marjal, en los que las características físico-químicas de las aguas son muy variables y aleatorias. La temperatura es máxima en agosto y mínima en febrero. Aquí $P$. zariquieye es el único Crustacea Decapoda presente, siendo la vegetación acuática muy abundante, principalmente constituida por $\mathrm{Po}$ tamogeton crispus, $P$. lucens, $P$. filiformis, MyriophyIlum verticillatum, Ceratophyllum demersum. C. sub- 


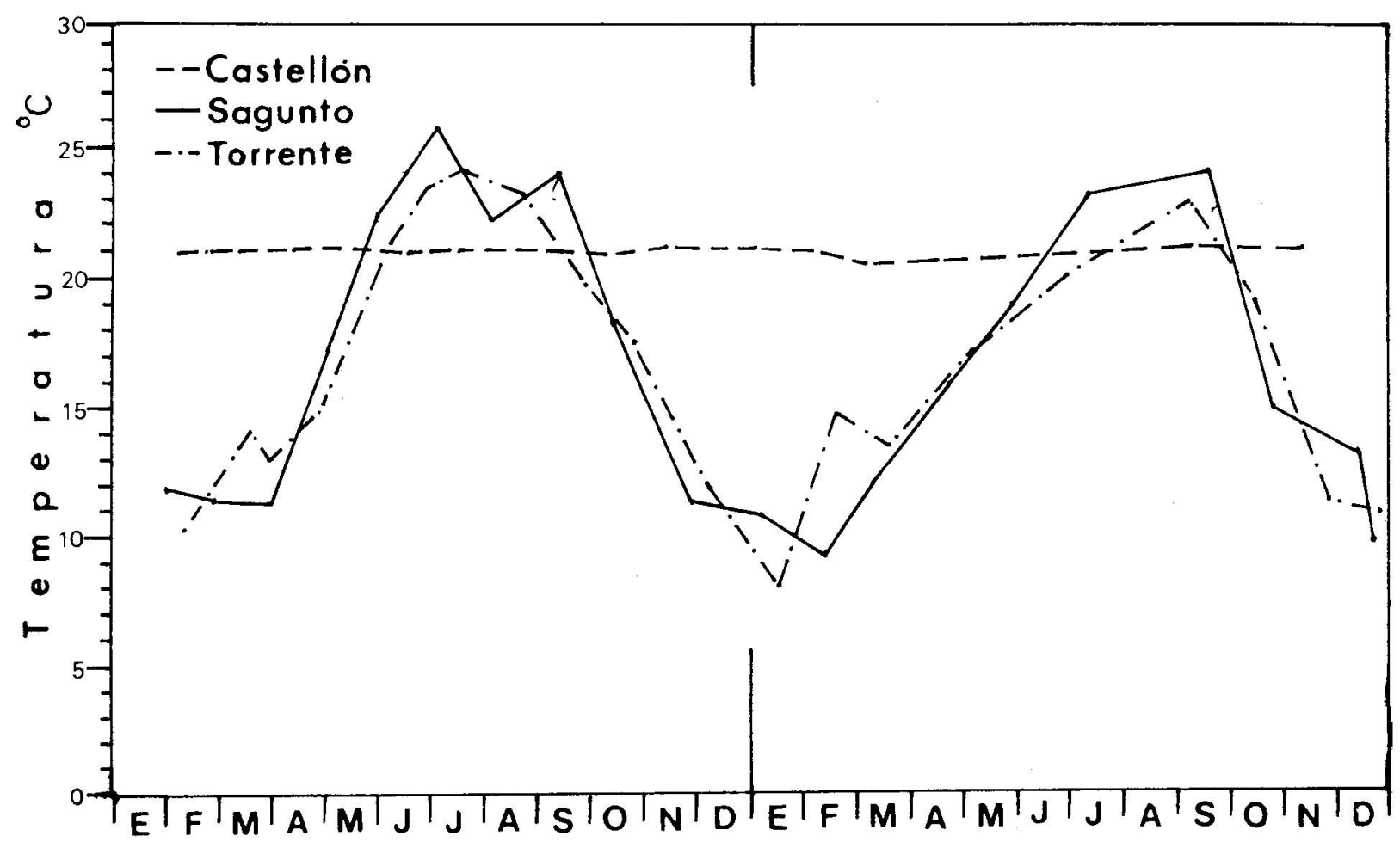

Figura 1.-Temperatura de las aguas en las tres localidades a lo largo de los dos años de estudio. Water temperatures in three localities through the study.

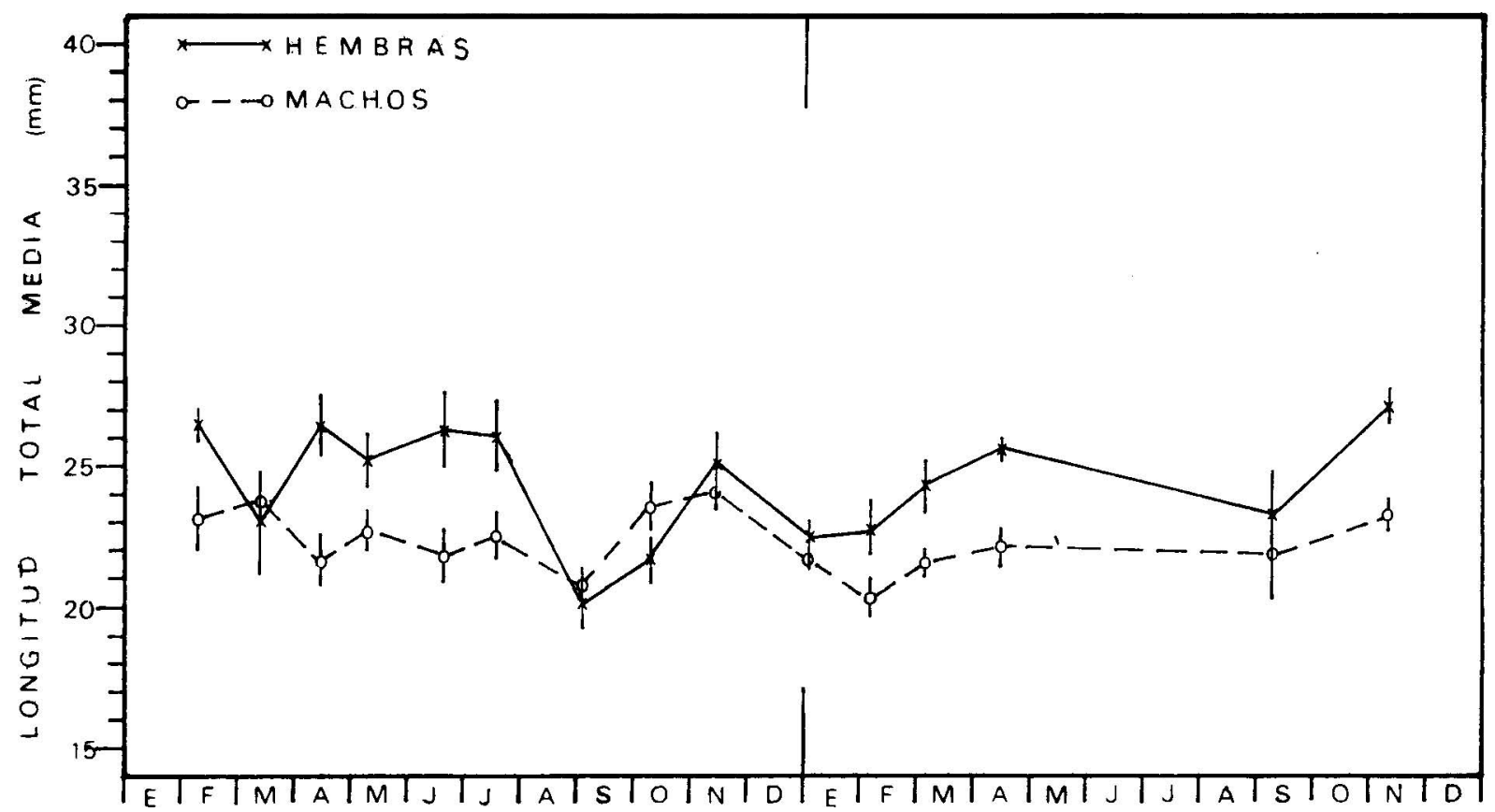

Figura ?.-Evolución de la población de Paleomonetes zariquieyi en la localidad de Castellón. Evolution of the population of Paleomonetes zariquieyi in place of Castellón. 
mersum, además de Lemna minor, Enteromorpha intestinalis y Rhizoclonium rigulare que se encuentran en las zonas remansadas de los canales. Resalta también la presencia de anguilas (Anguilla anguilla).

Por ultimo, el punto C (Torrente) es el más continental de los tres. Corresponde a unas charcas en el cauce de un barranco, donde las aguas están menos mineralizadas; y el ciclo anual de temperatura es análogo al del punto B (Figura 1). La vegetación acuática la constituye fundamentalmente Potamogeton natans; conviviendo aquí también Dugastella valentina (relación entre ambas especies de 1:1).

\section{MATERIAL Y METODOS}

Muestreo en el campo.- Los tres puntos fueron muestreados durante dos años consecutivos, con una perioricidad de alrededor de 40 días en cada uno de ellos; habiendose recogido un total de 53 muestras que comprenden un total de 4542 camarones.

Las capturas se llevaron a cabo mediante salabres, cuya malla de forma hexagonal, tenía una abertura de $1.0 \mathrm{~mm}$.. Estos salabres se rastreaban contra la vegetación, agitándola, recogiendose los ejemplares asi capturados en bandejas para su separación en frascos con agua del punto de captura para su transporte «in vivo» al laboratorio.

Análisis en el laboratorio. - Los camarones ya en el laboratorio, se separaban de otras especies que pudieran estar presentes, procediendose a la clasificación por sexos y a la determinación de su tamaño.

La diferenciación del sexo se llevó a cabo atendiendo a los caracteres sexuales de los dos primeros pares de pleópodos, anotándose en las hembras si estas estaban ovígeras, en cuyo caso se determinó el numero de huevos que transportaban en sus pleópodos y el tamaño de los mismos, o si la gónada estaba en desarrollo. En individuos jóvenes, al no tener claramente diferenciados sus caracteres sexuales secundarios en los pleópodos, es difícil llegar a precisar con total certitud el sexo; por lo que para homogeneizar los resultados hemos considerado como juveniles (sexo no determinado) a todos los camarones cuya longitud total era igual o inferior a $14.0 \mathrm{~mm}$., tamaño a partir del cual se aprecia ya el sexo con claridad.

La medida tomada a los camarones, siempre efectuada en fresco, fue la longitud total, considerada esta como la distancia entre el extremo distal del rostro y el extremo de las espinas del telson, estando el animal totalmente extendido y descansado todos ellos sobre el flanco derecho. Esta medida se llevó a cabo mediante un pie de rey cuando eran medianos o grandes y mediante lupa con ocular micrométrico cuando el tamaño de los camarones era pequeño, precisandose en todo caso hasta $\mathrm{O} .1 \mathrm{~mm}$..
El tamaño medio de la población de los camarones se estableció del valor medio de la longitud de los camarones de cada muestra, considerándose por separado para cada sexo.

\section{RESULTADOS}

\section{Ciclo biológico}

El ciclo biológico de Palaemonetes zariquieyi presenta características propias en cada una de las tres localidades estudiadas. En el punto A no se aprecia cambio marcado alguno a lo largo de los dos años de muestreo como se puede apreciar en la figura 2; mientras que en los puntos $\mathrm{B}$ y $\mathrm{C}$ se establece con claridad un ciclo anual, con un mínimo del tamaño medio de los individuos de la población durante el mes de julio, época en la que se incorporan los camarones de la nueva generación (figuras 3 y 4).

Algunos pocos individuos de la vieja generación sobreviven hasta los meses de octubre-noviembre en el punto B (figura 3); mientras que en $\mathrm{C}$, algunos llegan a perdurar durante el invierno siguiente.

\section{Periodo de reproducción}

En el punto A no existe un periodo concreto de reproducción de esta especie, ya que se detecta la presencia de hembras ovigeras en la población prácticamente a lo largo de todo el año (figura 5). Por el contrario, en B y C la época en que aparecen las hembras ovígeras es en primavera, como se puede apreciar en los histogramas de las figuras 6 y 7 ; y que con detalle se expone en la figura 8. Coincide ello cuando las aguas en estas dos localidades alcanzan una temperatura entre 15 y $18^{\circ} \mathrm{C}$.

En Sagunto se observa la aparición de hembras ovigeras en el mes de abril; mientras que en Torrente la aparición de hembras ovígeras es ligéramente más tardía. En ambas localidades, a mediados del mes de julio, aproximadamente el $90 \%$ de la población de camarones está constituida por individuos de la nueva generación.

Existen hembras que a pesar de estar ovígeras, aun se puede observar en ellas un cierto desarrollo de su gónada, señal inequívoca de que efectuarán una segunda puesta durante el verano; aunque quizás es mejor estimar que la puesta de los huevos tiene lugar de un modo gradual y en función de la gradual maduración de los ovocitos. Ello vendría a explicar la presencia de hembras ovígeras incluso después del periodo central de reproducción, tal como se puede apreciar en la figura 6 , en donde se comprueba la presencia de alguna de estas hembras durante el verano. Un hecho semejante ha sido descrito en $\mathrm{P}$. argentinus (Goldstein y Lauria de Cidre, 1974).

Crecimiento

En la localidad de Castellón, el crecimiento de los 


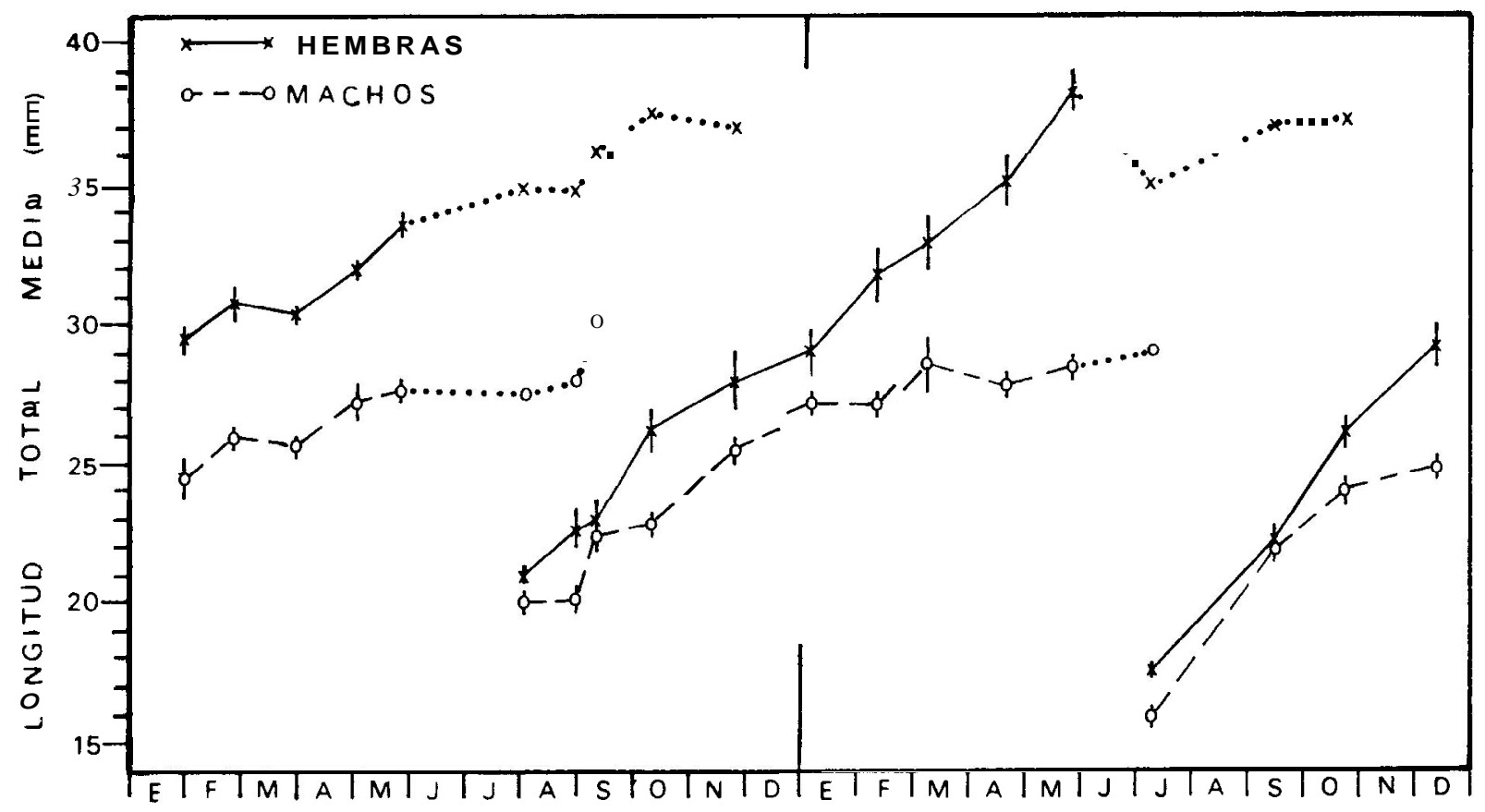

Figura 3.-Evolución de la población de camarones en la localidad de Sagunto. Mediante puntos se viene a representar a los individuos de la vieja generación.

Life cycle of the shnmp P.zariquieyi in place of Sagunto. Older generation represented with a dotted line.

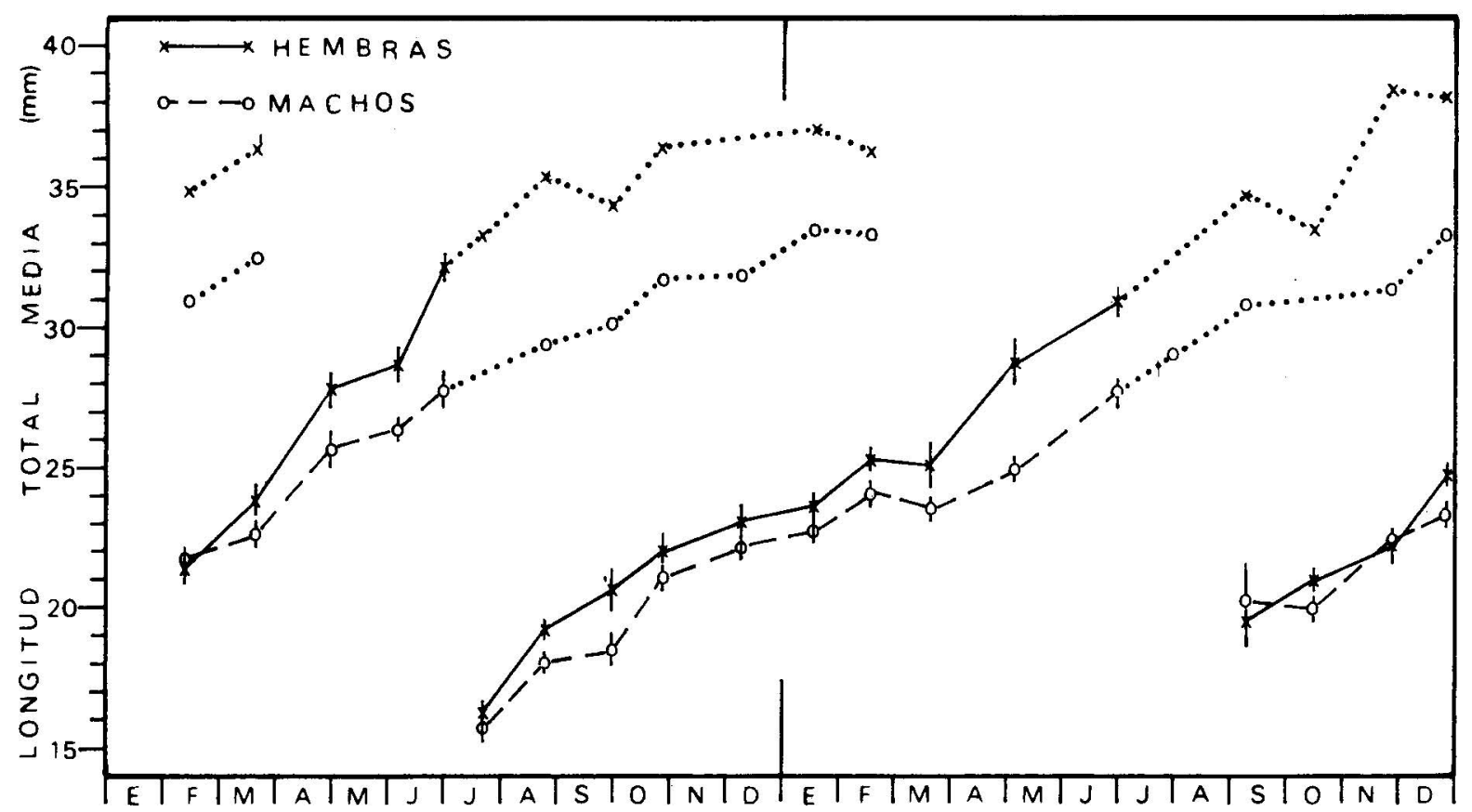

Figura 4.-Evolución de la población de camarones en Torrente. Los individuos de la vieja generación están representados por la línea de puntos, siempre representados estos en la población por escaso numero de ejemplares.

Life cycle of P. zariquieyi in place of Torrente. Older generation represented with a dotted line. 
Tabla 1.-Características físico-químicas (máximoy mínimo) de las aguas en las tres localidades de estudio. Physical and chemical conditions-maximum and minimum-of the waters during the study period.

LOCALIDAD

(A) Castellón

(B) Sagunto

(C) Torrente

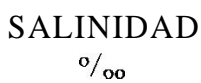

$$
\begin{aligned}
& 1.1-0.7 \\
& 7.9-1.0 \\
& 0.8-0.5
\end{aligned}
$$

DUREZA TOTAL od.
DUR. CARBONATOS ${ }^{\circ} \mathrm{d}$.
CLORUROS mgr./1

$400-300$

$6.000-100$

$390-130$

Tabla 2.-Longitud máxima medida (mm.)en los camarones de las tres localidades estudiadas. Se expone el tamaño de los individuos mayores de cada sexo.

Maximum sizes, in mm. measured in the three places studies, showing the two largest specimens ofeach sex.

CASTELLON

$39.7-39.0$

$31.5-31.4$
SAGUNTO

$45.7-45.3$

$35.2-34.4$
TORRENTE

$42.8-42.0$

$35.9-35.5$

Tabla 3.-Porcentaje de hembras en cada una de las tres poblaciones de estudio. Percentage of females in each of the studies populations.

\section{LOCALIDAD}

(A) Castellón

(B) Sagunto

(C) Torrente

\section{NUMERO DE CAMARONES}

849

1.757

1.936
PORCENTAJE DE HEMBRAS
$50.3 \%$
$40.1 \%$
$49.3 \%$

Tabla 4.-Númerode huevos transportados por las hembras ovígeras. Number of eggs carried by the ovigerous females.

\section{LOCALIDAD}

(A) Castellón

(B) Sagunto

(C) Torrente

\section{MINIMO}

\section{6}

14

14
MAXIMO

55
126
72

MEDIA

32

61

37 camarones a lo largo del año no puede concretarse (fjgura 2) al no existir un periodo delimitado de reproducción. En las otras dos localidades el crecimiento de los camarones es rápido al principio de su ciclo biológico (meses de verano), para luego menguar, volviendose otra vez acusado coincidiendo con el final de su ciclo (figuras 3 y 4 ).

Las hembras alcanzan un tamaño significativamen- te mayor $(P=0.01)$ que los machos. Esto se observa en las localidades de Sagunto y Torrente; mientras que en la localidad de Castellón ello no queda totalmente claro debido al constante aporte de juveniles a esta población, enmascarándose esta diferencia. En la tabla 2 se exponen los dos tamaños máximos medidos en este estudio en cada uno de los tres puntos y para cada sexo. Se puede apreciar el distinto tamaño que 


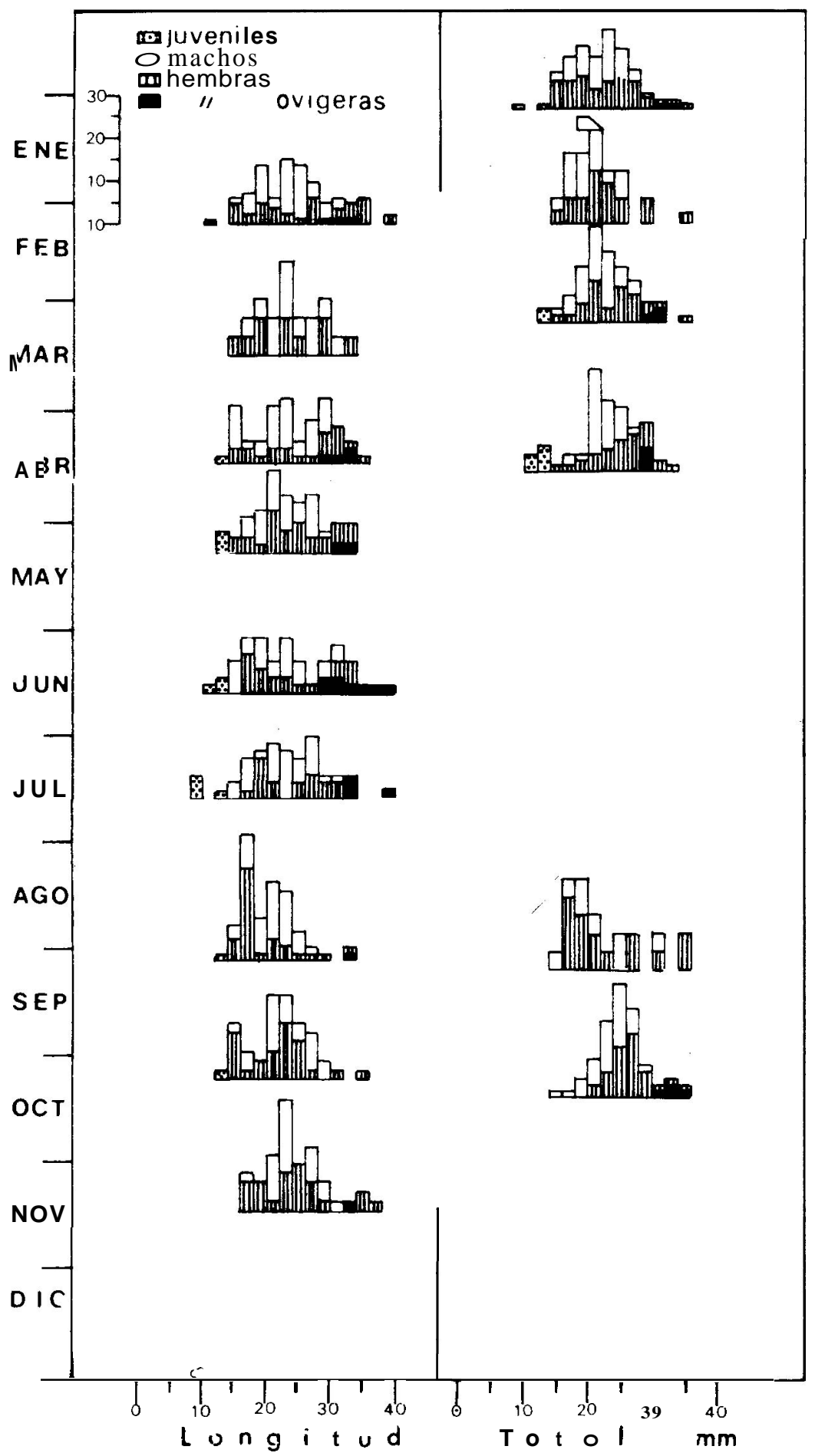

Figura 5.-Histogramas de frecuencia de individuos en la localidad de Castellón.Obsérvese que en casi la totalidad de meses existen hembras ovigeras.

Frecuency of the size classes of the samples in place of Castellón. Almost the totality of months there are females breeding. 


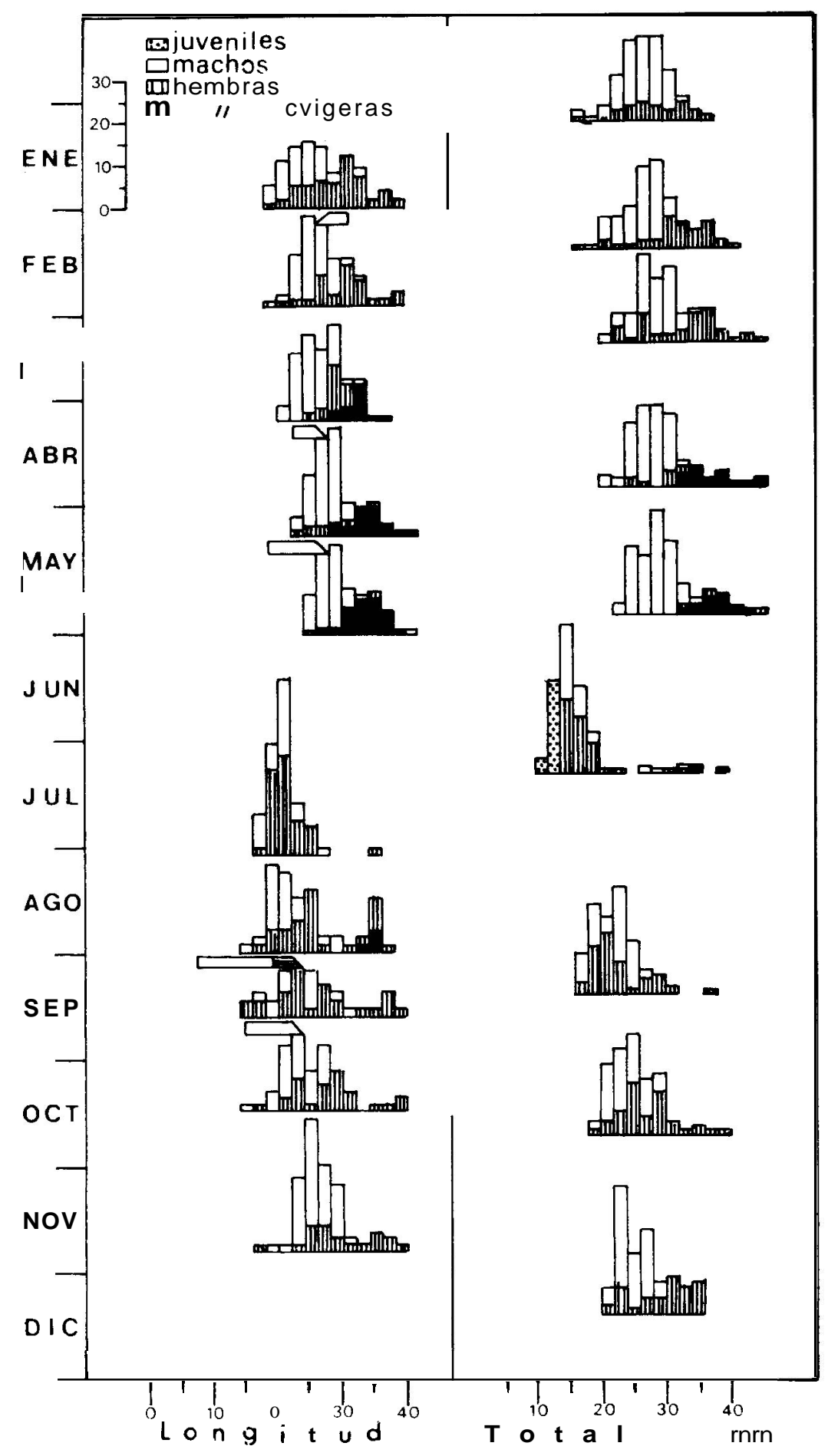

Figura 6.-Histogramas de frecuencia de los individuos de P. zariquieyi en la localidad de Sagunto. Frecuency of the size classes of the samples in place of Sagunto. 


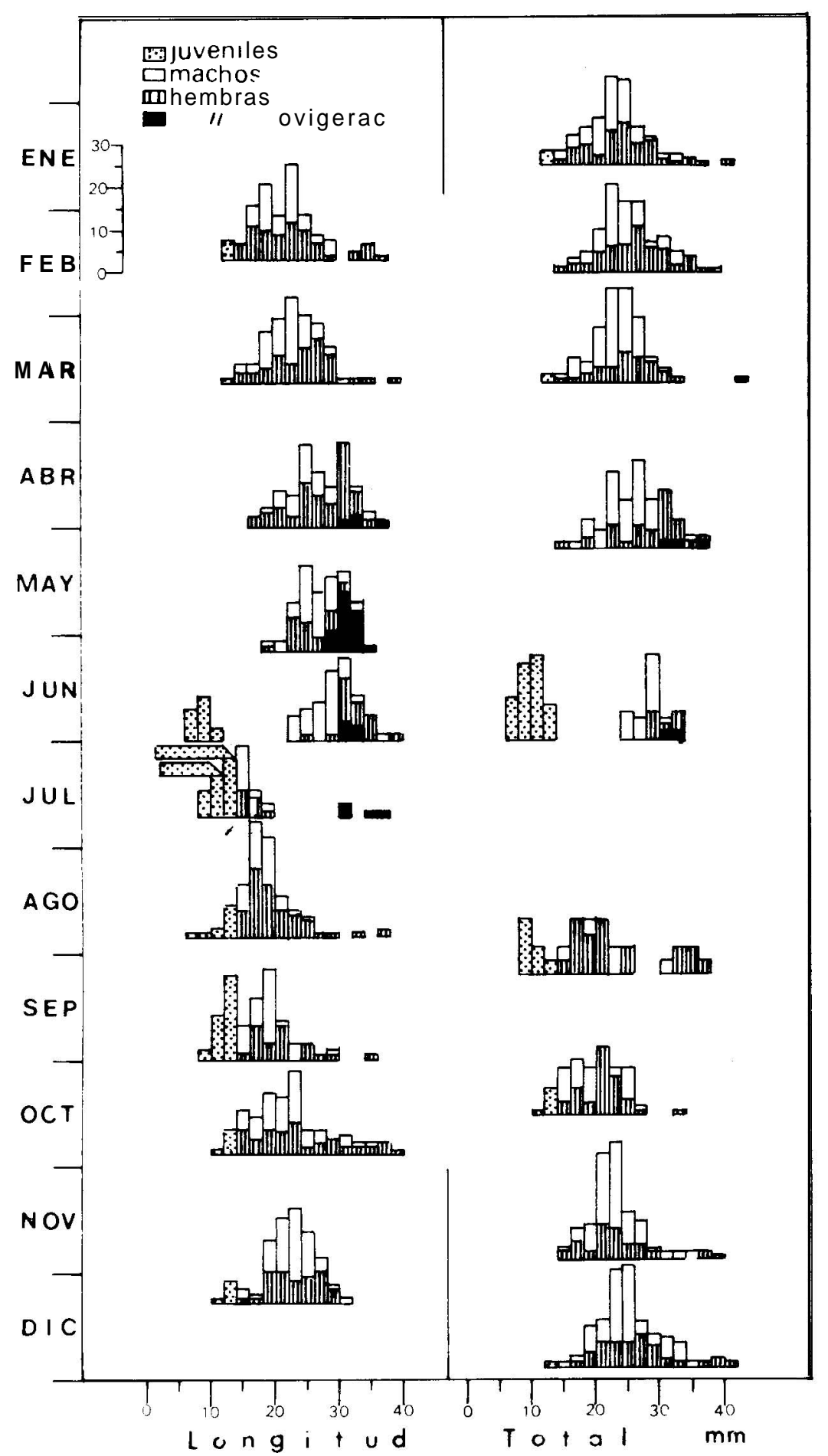

Figura 7.-Histogramas de frecuencia de los individuos de P. zariquieyi en la localidad de Torrente. Frecuency of the size classes of the samples in place of Torrente. 
los camarones alcanzan en cada una de las localidades, atribuibles las diferencias a las distintas condiciones ecológicas.

Proporción de sexos

En la tabla 3 se expone el porcentaje de hembras en cada una de las localidades. Tanto en Castellón como en Torrente la relación entre sexos es de 1:1; mientras que en Sagunto los machos son predominantes $(3: 2)$

Fecundidad

Palaemonetes zariquieyi es un Palaemonidae de la serie macrogenitor (Sollaud, 1923), puesto que sus huevos tienen un tamaño medio de $1.53 \pm 0.12 \mathrm{~mm}$. en su eje mayor y de $1.22 \pm 0.10$ en el eje perpendicular al anterior. De aquí que el número de huevos que viene a transportar la hembra ovígera en sus pleópodos en muy pocos ejemplares supere el centenar.

En la tabla 4 se muestra el número de huevos transportados por las hembras ovígeras. tanto en su valor medio, como el máximo y mínimo en las tres localidades. Las diferencias son atribuibles al distinto tamaño que alcanzan los camarones en cada una de estas poblaciones, acorde con las diferencias de crecimiento ya indicadas; y que gráficamente se expone en la figura 9.

El número de huevos transportados por las hembras ovígeras se expone en la figura 10. estando correlacionado el tamaño de la puesta con el de las hembras $(r=0.7076 ; n=250)$. No obstante, se comprueba en esta figura una cierta dispersión de los valores; y tal como se indicaba al señalar el periodo de reproducción de esta especie, cabe atribuir dicha causa a que existen hembras ovígeras que no vacían totalmente su gónada en una pista. De aquí, si el cálculo de la relación del número de huevos de la puesta con la longitud total de los camarones se efectua considerando tan solo aquellas tres hembras que presentan el máximo de huevos dentro del rango de tamaño de 1.0 mm., el coeficiente de correlación es entonces de $r=0.9541(n=35)$.

\section{DISCUSION}

Las diferencias en el ciclo biológico de Palaemonetes zariquieyi en las tres localidades estudiadas hay que atribuirlas a las características ambientales que presentan los distintos puntos. Hofer et al. (1980) vinieron a mostrar diferencias, especialmente en su tamaño, de tres poblaciones de P. antennarius del lago Garda, indicando que existía una correlación negativa entre la contaminación de las aguas y el tamaño medio de los camarones, además de que la temperatura es un factor determinante en su supervivencia.

En el punto A (Castellón) el ciclo biológico de $P$. zariquieyi no se constata debido a que las condiciones ambientales son constantes, particularmente la temperatura, lo que explicaría la presencia de hembras ovigeras prácticamente a lo largo de todo el año. Beck \& Cowell (1976) señalaron que Palaemonetes paludosus varía su periodo de reproducción con la latitud, indicando que dicho periodo de reproducción es más amplio en puntos más meridionales, en los que cabe esperar que las temperaturas de las aguas sean más cálidas y oscilen menos. Así Dobkin (1963) detectó la presencia de hembras ovígeras de $P$. paludosus todo el año en Miami (U.S.A.).

En las localidades de Sagunto y Torrente la irrupción de hembras ovígeras en la población acontece durante la primavera; pero en Torrente la epoca de reproducción es ligeramente más tardía que en Sagunto, donde la temperatura de las aguas es algo más elevada a lo largo del año. En base a este factor hay que interpretar las diferencias en el periodo de reproducción observadas en otras especies de Palaemonetes, como se deduce de los datos de Wood (1967) para con P. pugio; de Knowlton \& Williams (1970) para con $P$. pugio y $P$. vulgaris; de Meehean (1936), de White (1949) y de Nielsen \& Reynolds (1977) para con P. kadiakensis; de Hedgepeth (1950) para con $P$. intermedius; y que especialmente queda ello más realzado para $P$. argentinus que vive en el hemisferio austral, con un periodo de reproducción que se extiende desde agosto hasta febrero (Goldstein \& Lauria de Cidre, 1974) cuando allí lógicamente en aquel hemisferio coincide con los meses de primavera-verano.

De los datos del presente estudio se deduce que $P$. zariquieyi es una especie de ciclo biológico anual; e igualmente un ciclo anual ha sido descrito para $P$. paludosus (Beck \& Cowell, 1976); P. kadiakensis (Meehann, 1936; y Nielsen \& Reynolds, 1977); y $P$. pugio (Welsh, 1975). Estos autores también resaltan el que algunos pocos individuos de estas especies puede que alcancen a vivir el invierno posterior al de su periodo de reproducción, al igual como sucede para con $P$. zariquieyi, especialmente en la localidad de Torrente.

El crecimiento de $P$. zariquieyi no se detiene a lo largo de su ciclo biológico, ni tan siquiera durante los meses invernales, cuando la temperatura de las aguas son más bajas. Welsh (1975) señaló un crecimiento similar en P. pugio; pero indicando que en los meses centrales de su ciclo (invierno) el crecimiento era prácticamente nulo. En el punto B (Sagunto) el tamaño medio alcanzado por los camarones es mayor que en las otras dos localidades, aspecto que hay que atribuir a las condiciones tróficas allí existentes, con gran diversidad de vegetación, habitat natural de estos camarones.

Las hembras de $P$. zariquieyi alcanzan un tamaño significativamente mayor que los machos, al igual 

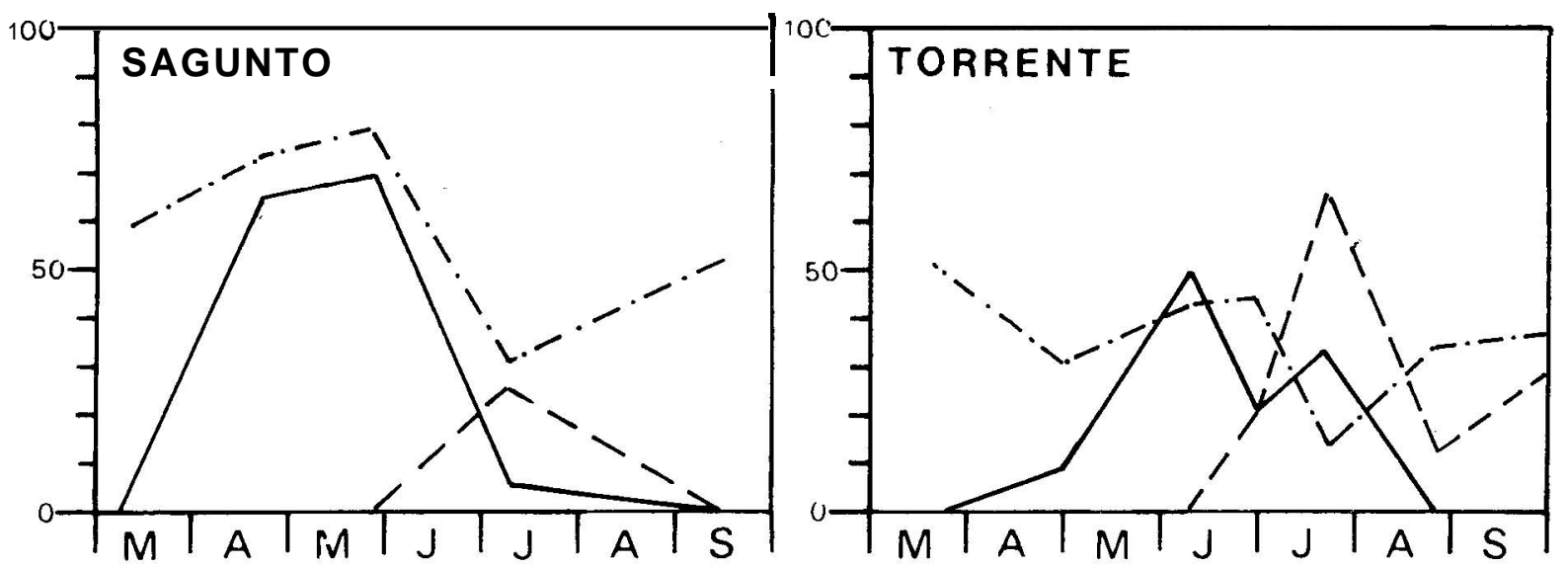

Figura 8.-Composición de las poblaciones de P. zariquieyi en las localidades de Sagunto y Torrente durante el periodo de reproducción (--) porcentaje de machos; $(\rightarrow$ porcentaje de juveniles; (-)porcentaje de hembras ovígeras del total de hembras.

Composition of the population of P. zariquieyi in places of Sagunto and Torrente dunng the breeding season. (-.-) males percentage; $(-)$ juveniles percentage; $(-)$ breeding females percentage of the total of females.
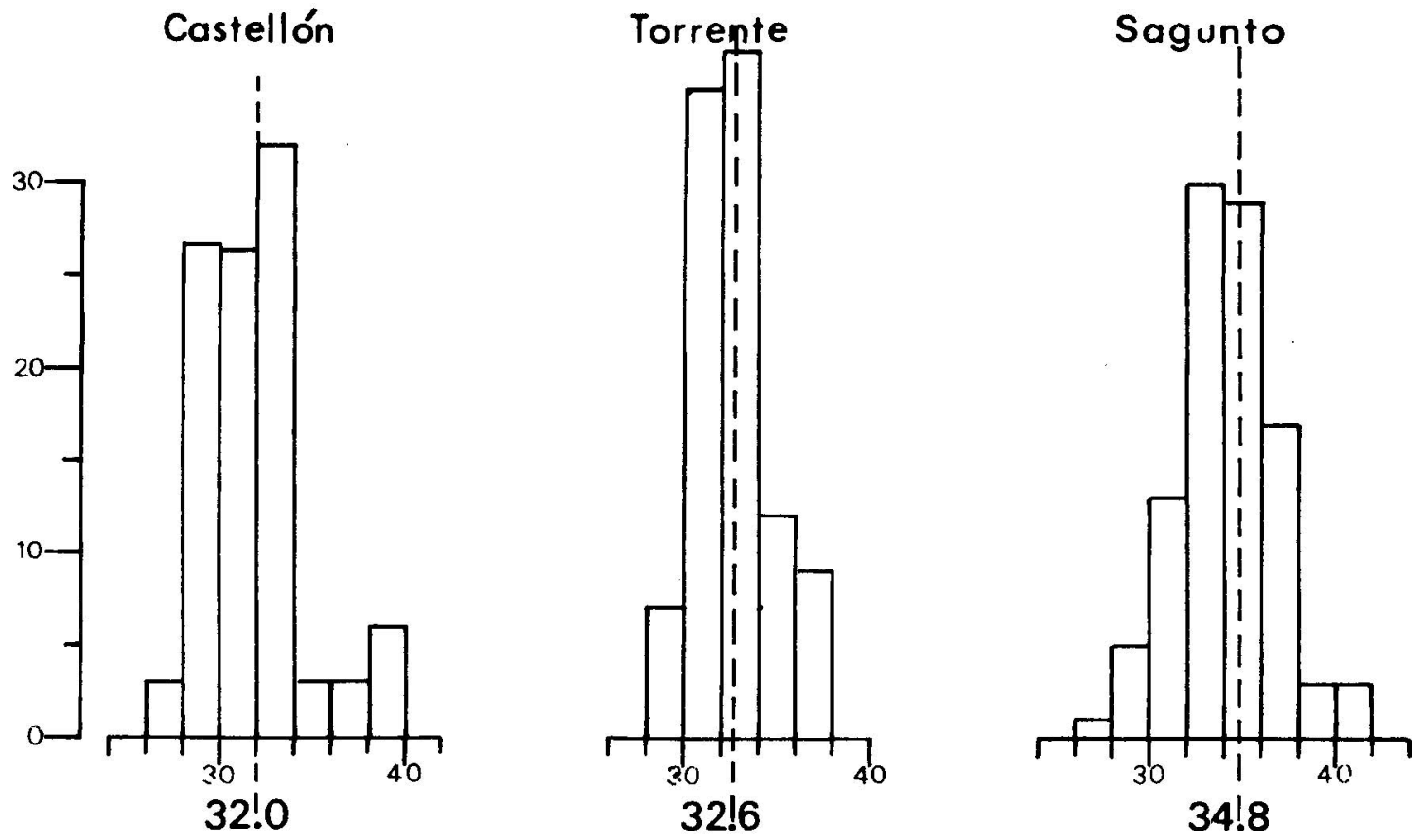

Figura 9.-Histogramas de frecuencias de tamaño de las hembras ovígeras en cada una de las tres localidades de estudio. Se estudia el tamaño medio de estas hembras (mm.).

Frecuency ofthe size classes of the breeding females in the three places. Mean these females is showed. 


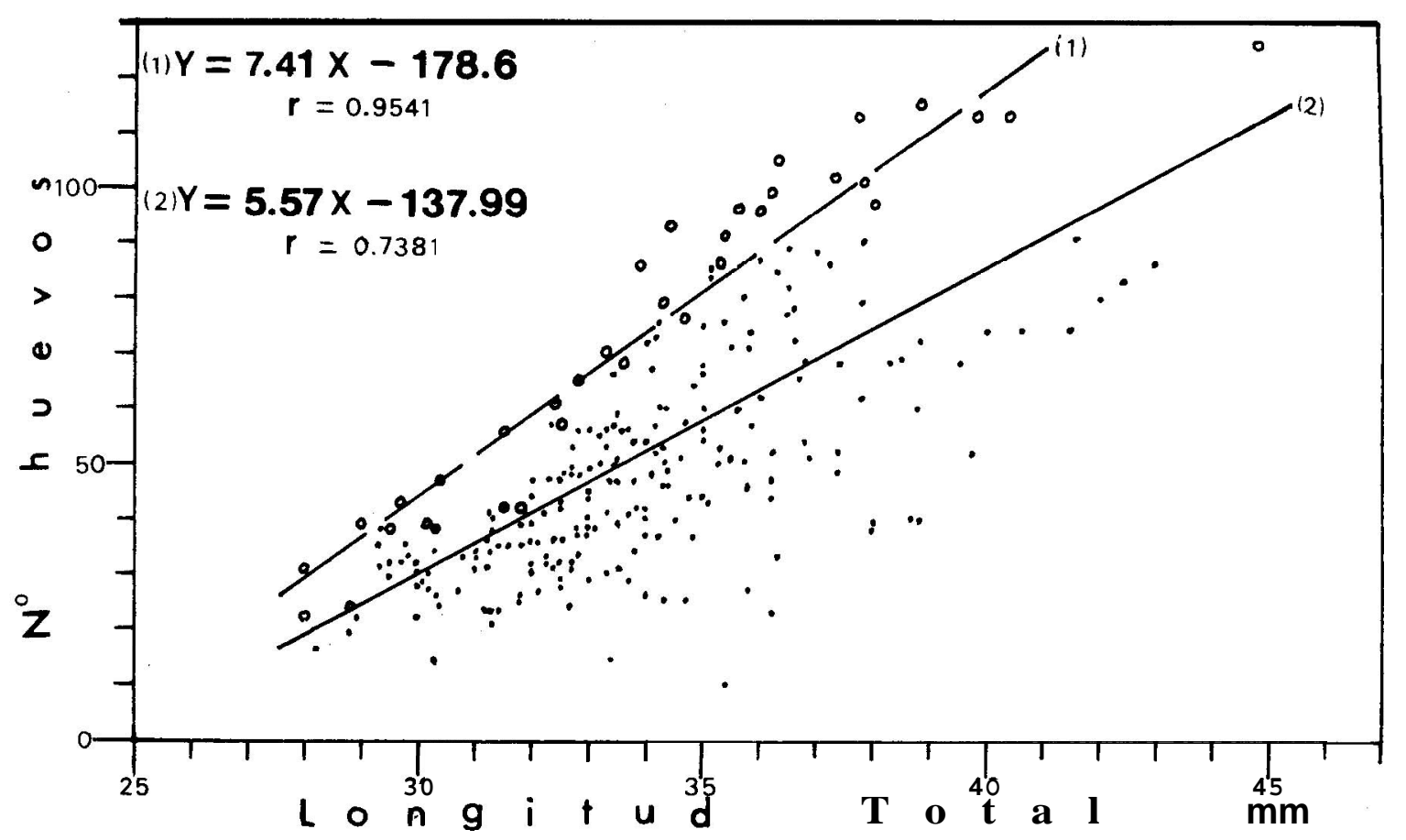

Figura 10.-Relación entre el numero de huevos y la longitud total en las hembras ovígeras, considerando la totalidad de ellas (2) y tan solo a las que presentan el máximo de huevos dentro de cada intervalo de tamaño de $1.0 \mathrm{~mm}$. (1). Estas se representan en el gráfico mediante un circulo en blanco (o).

Ralationship number of eggs and total length of the ovigerous females.

que ocurre en P. pugio (Welsh, 1975), P. paludosus (Beck \& Cowell, 1976), en P. kadiakensis (Nielsen \& Reynolds, 1977), y en P. antennarius (Hofer et al., 1980). Ello no se aprecia de las muestras de CasteIlón, debido a las peculariedades de esta población, en la que constantemente existen juveniles incorporándose a la misma.

El número de huevos transportados en los pleópodos por la hembra ovígera de P. zariquieyi está correlacionado con su tamaño, aunque al haber hembras que efectuan la puesta en dos etapas y en función de la gradual maduración de los ovocitos, explica ello el que haya hembras que no presenten en sus pleópodos el número de huevos que cabría esperar por su tamaño; característica esta que también ha sido observada en P. argentinus (Goldstein \& Lauria de Cidre, 1974).

En los puntos de Castellón y Torrente existe igualdad en la proporción de sexos (1:1). Nielsen \& Reynolds (1977) encontraron relaciones distintas en tres poblaciones estudiadas por ellos de P. kadiakensis: señalando Beck \& Cowell (1976) el predominio de las hembras sobre los machos en $P$. paludosus. Por su parte Welsh (1975) indicó el predominio de los machos (3:2) sobre las hembras en P. pugio durante al- gunos meses de su ciclo biológico, atribuyéndolo a que según Nixon \& Oviatt (1973) los «Killifishes» (Fundulus sp.) predan sobre los camarones de mayor tamaño que son las hembras ovigeras, las cuales tienen unos movimientos más torpes. debido a los huevos que transportan en sus pleópodos. Quizás sea algo similar lo que le ocurre a P. zariquieyi en la localidad de Sagunto (relación entre los machos y las hembras de 3:2), puesto que habitan allí anguilas (Anguilla anguilla) las cuales en la región de estudio se alimentan entre otras presas de camarones (Gandolfi, 1917).

\section{BIBLIOGRAFIA}

Beck, J.T. y B.C. Cowell 1976.- Life history and ecology of the freshwater caridean shrimp, Palaemonetes paludosws (Gibbes).-Am. Midl. Nat. 98: 52-65.

Dobkin, S. 1963.- The larval development of Palaemonetes paludosus (Gibbes. 1850) (Decapoda. Palaemonidae) reared in the laboratory.- Crustaccana. 6 : $41-61$. 
Gandolfi, A. 1917.- Algunas observaciones sobre la alimentación de la anguila de la Albufera de Valencia, Santander, Lisboa y Aveiro.- An. Inst. Gen. Tec. Valencia, 2(6): 1-6.

Goldstein, B. Y L. Lauria de Cidre 1974.- Ciclo de maduración sexual y observaciones preliminares sobre el desove del camarón dulceacuicola Palaemonetes argentinus (Nobili, 1901). 1. Hembra.- Physis secc. B Aguas Cont. Org. 33(87): 165-176.

Hedgepeth, J.W. 1950.- Notes on the marine invertebrate fauna of salt flat areas in Aransas National Wildlife Refuge, Texas.- Publ. Inst. Mar. Sci., 1: 103-119.

Hofer, R., J. Dalla Via y B. Obermuller 1980.- Okologiske untersuchgen an Palaemonetes antennarius im Gardasee.- Arch. Hydrobiol., 90(2): 197-209.

Holthuis, L. B. 1978.- Decapoda. In Limnofauna Europea (ILLJES, J. ed.): 188-193.- Gustav Fischer Verlag. New York.

Knowlton, R.E. y A.B. Willians 1970.- The life history of Palaemonetes vulgaris and Palaemonetes pugio in coastal Nort-Carolina.- J. Elish. Mitch. Sci. Soc., 86(4): 185.

Margalef, R. 1953.-Los crustáceos de las aguas continentales ibéricas.- Dirección Gen. Montes. Ministerio de Agricultura. Madrid, 243 págs.

Meehean, O.L. 1936.- Notes on the freshwater shrimp, Palaemonetes paludosus (Gibbes).- Trans. Am. Microsc. Soc., 55: 433-441.

Nielsen, L.A. y J.B. Reynolds 1977.- Population characteristics of a freshwater shrimps, Palaemonetes Kadiakensis Rathbun.- Trans. Missouri Acad. Sci., 10 \& 11: 44-57.
Nixon, S.W. y C.A. Oviatt 1973.- Ecology of a New England salt marsh.- Ecol. Monogr., 43: 463-498.

Sanz, A. 1980.- biología y ecología de Palaemonetes zariquieyi Sollaud, 1939 (Crustacea, Decapoda, Palaemonidae).- Tesis doctoral. Universidad de Valencia. (no publicada).

Sanz, A. 1983.- Localidades y sus características ambientales del camarón Palaemonetes zariquieyi Sollaud, 1939 (Crustacea, Decapoda).- Actas I Congr. Ibérico Entomologia: 737-742.

Sollaud, E. 1923.- Le development larvaire des «Palaemoninae».- Bull. Biol. France-Belg.. 57(4): 509-603.

Sollaud, E. 1939.- Sur un Palaemonetes endemique, $P$. Zariquieyi, n. sp., loca lisé dans la plaine littorale du Golfe de Valence.- Trav. Sta. Zool. Wimereux, 13: 635-645.

Welsh, B.L. 1975. The role of grass shrimp, Paleomonetes pugio, in a tidal marsh ecosystem. Ecology, 56: 513-530.

White, F.A. 1949. Preliminary notes on the breeding season of Paleomonietes kadiakensis Rathbun inthe Baton Rouge area. Proc. La. Acad. Sci. 12: 71-74.

Wood, C.E. 1967. Physioecology of the grass shrimp Paleomonetes pugio, in Gaveston Bay estuarine system. Contrib. Mar. Sci., 12: 54-79.

Zariquiey, R. 1946. Crustáceos Decápodos mediterráneos. inst. Esp. Est. Mediterráneos. Barcelona, 181 PP

Zariquiey, R. 1968. Crustáceos Decápodos Ibéricos. Inv. Pesq. 32: 510. pp. 